\title{
Affine quantization of $\left(\varphi^{4}\right)_{4}$ succeeds while canonical quantization fails
}

\author{
Riccardo Fantoni $\circledast^{1, *}$ and John R. Klauder $\circledast^{2, \dagger}$ \\ ${ }^{1}$ Università di Trieste, Dipartimento di Fisica, Strada Costiera 11, 34151 Grignano (Trieste), Italy \\ ${ }^{2}$ Department of Physics and Department of Mathematics University of Florida, \\ Gainesville, Florida 32611-8440, USA
}

(Received 15 December 2020; accepted 25 March 2021; published 22 April 2021)

\begin{abstract}
Covariant scalar field quantization, nicknamed $\left(\varphi^{r}\right)_{n}$, where $r$ denotes the power of the interaction term and $n=s+1$ where $s$ is the spatial dimension and 1 adds time. Models such that $r<2 n /(n-2)$ can be treated by canonical quantization, while models such that $r>2 n /(n-2)$ are nonrenormalizable, leading to perturbative infinities, or, if treated as a unit, emerge as 'free theories'. Models such as $r=2 n /(n-2)$, e.g., $r=n=4$, again using canonical quantization also become 'free theories', which must be considered quantum failures. However, there exists a different approach called affine quantization that promotes a different set of classical variables to become the basic quantum operators and it offers different results, such as models for which $r>2 n /(n-2)$, which has recently correctly quantized $\left(\varphi^{12}\right)_{3}$. In the present paper we show, with the aid of a Monte Carlo analysis, that one of the special cases where $r=2 n /(n-2)$, specifically the case $r=n=4$, can be acceptably quantized using affine quantization.
\end{abstract}

DOI: 10.1103/PhysRevD.103.076013

\section{INTRODUCTION}

The family of covariant scalar field theories under consideration have classical Hamiltonians given by

$$
\begin{aligned}
H(\pi, \varphi)= & \int\left\{\frac{1}{2}\left[\pi(x)^{2}+(\vec{\nabla} \varphi)(x)^{2}+m^{2} \varphi(x)^{2}\right]\right. \\
& \left.+g \varphi(x)^{r}\right\} d^{s} x,
\end{aligned}
$$

where the mass $m>0$, the coupling constant $g \geq 0, r$ is the power of the interaction term, and $s$ is the number of spatial dimensions. As classical elements they lead to suitable equations of motion and these solutions automatically guarantee that, for $T>0$, all such solutions obey the rule that $\int_{0}^{T} \int H(\pi(x, t), \varphi(x, t)) d^{s} x d t<\infty$.

However, acceptable classical solutions do not tell the whole story. The domain of the example in (1) includes the complete set of arbitrary continuous paths, $\pi(x, t)$ and $\varphi(x, t)$, that determine the domain

$\mathcal{D}(\pi, \varphi)=\left\{\int_{0}^{T} \int H(\pi(x, t), \varphi(x . t)) d^{s} x d t<\infty\right\}$.

*riccardo.fantoni@posta.istruzione.it

†klauder@phys.ufl.edu

Published by the American Physical Society under the terms of the Creative Commons Attribution 4.0 International license. Further distribution of this work must maintain attribution to the author(s) and the published article's title, journal citation, and DOI. Funded by SCOAP.
This expression for the domain is unchanged if the interaction term is excluded, while, on the other hand, the given domain will be dramatically reduced from the true free theory domain if the interaction term is sufficiently strong and has been introduced. These questions can be answered if we show the domains may be studied.

\section{A. Review of principal domains}

The term $\pi(x, t)^{2}$ is equal to $\dot{\varphi}(x, t)^{2}$, and if we include $t$ as just another spatial variable the classical Hamiltonian becomes

$H(\varphi)=\int\left\{\frac{1}{2}\left[(\vec{\nabla} \varphi)(x)^{2}+m^{2} \varphi(x)^{2}\right]+g \varphi(x)^{r}\right\} d^{s} x$,

where now $x=\left(x_{0}, x_{1}, x_{2} \ldots, x_{s}\right)$, where $x_{0}=c t$ and $n=s+1$. The contribution of the separate terms can be captured if we observe that the three following cases must be treated separately and constitute unitarily inequivalent quantizations,

$\left\{\int \varphi(x)^{r} d^{n} x\right\}^{2 / r}<$ or $=$ or $>\int\left\{(\vec{\nabla} \varphi)(x)^{2}+m^{2} \varphi(x)^{2}\right\} d^{n} x$.

The domain of this expression consists of all $\{\phi(x)\}$ for which $H(\phi)<\infty$. For a given finite spacetime dimension $n$ and power $r$, it is of considerable interest to learn if the domain changes when the interaction coupling constant changes from $g=0$ to $g>0$. The study of that issue for all 
$\{\phi(x)\}$ involves an explicit expression in which $t$ becomes an additional "spatial variable"

$$
\left\{\int \phi(x)^{r} d^{n} x\right\}^{2 / r}=C \int\left[(\vec{\nabla} \phi)(x)^{2}+m^{2} \phi(x)^{2}\right] d^{n} x .
$$

If $C$ is bounded above for all $\phi$, then the domain of $H$ is the same with $g=0$ or $g>0$. If $C=\infty$ then the domain of $H$ is larger when $g=0$ than it is when $g>0$. It follows $[1,2]$ that the domain does not change provided that $r<2 n /(n-2)$. If, on the other hand, $r>2 n /(n-2)$, then the domain of $H$ drops as soon as $g>0$, and thus when one seeks the domain in the limit that $g \rightarrow 0$, continuity forces the resultant domain to be the smaller domain.

The focus hereafter, in this paper, is on the case $r=$ $2 n /(n-2)$, and more explicitly, we choose $r=n=4$. $^{1}$ This model has been studied by applying canonical quantization, and the universally accepted result [3-5] is that this model becomes a "free model" despite the presence of the interaction and $g>0$. Such a result may be considered as a quantum failure. Additional efforts has been made in Ref. [6] to quantize $\left(\phi^{4}\right)_{4}$ models using alternative procedures that, regrettably, have also led only to a "free theory."

Affine quantization can account for certain classical models that experience two different classical domains, one (free) without an interaction term, $g=0$, and one (nonfree) with the interaction term, $g>0$. If necessary, such models automatically choose the smaller domain as $g \rightarrow 0$ by simple continuity. ${ }^{2}$ This feature can imply either that the quantization leads only to the free theory, or the quantization leads to the form of a nonfree theory. As an example, canonical quantization of $\left(\varphi^{4}\right)_{4}$ leads only to a free theory [3-5], which, incidentally, may lead to unexpected difficulties in the Standard Model (SM). Our quantization of $\left(\varphi^{4}\right)_{4}$ by affine quantization is a procedure that is just like canonical quantization, after choosing a different pair of classical variables to promote to operators, and can fit well with other quantization procedures, as is implicit in our study.

Affine quantization [8] has offered positive results for several models, one of which includes the nonrenormalizable model $\left(\varphi^{12}\right)_{3}$ [9]. In this paper we seek to find out whether affine quantization will lead to a positive quantization of the model $\left(\varphi^{4}\right)_{4}$, or, if not positive, what behavior is actually found.

\section{CANONICAL AND AFFINE QUANTIZATION OF THE SCALAR EUCLIDEAN FIELD THEORY}

Canonical quantization has been the go to method of quantization for many decades. Clearly, the reason for this loyalty is because its use has been so successful. The simple

\footnotetext{
${ }^{1}$ The only other similar model is $r=6, n=3$.

${ }^{2}$ The paper [7] using ultralocal field models clearly illustrates how the smaller domain wins.
}

examples from quantum mechanics have led to similar procedures when dealing with field quantization. The formulations of continuous fields can also lead to unwanted divergences. This behavior invites the use of a formal analysis, which addresses selected equations as beacons that regularization of the procedure enables preliminary analysis to occur. This section is devoted to a formal analysis of both canonical and affine quantization of fields initially guided by elementary examples.

For a single classical degree of freedom, the favored variables $-\infty<p, q<\infty$ and a Poisson bracket $\{q, p\}=1$ lead to the basic quantum operators $P, Q$, which obey

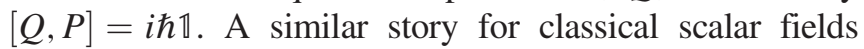
$-\infty<\pi(x), \varphi(x)<\infty$ has a Poisson bracket $\{\varphi(x)$, $\pi(y)\}=\delta^{s}(x-y)$ which points toward basic quantum operators $\hat{\pi}(x), \quad \hat{\phi}(x), \quad$ that obeys $[\hat{\phi}(x), \hat{\pi}(y)]=$ $i \hbar \delta^{s}(x-y)$. Ideally, the semiclassical action functional for a field theory model is given by

$S^{(c)}[\varphi]=\int\left\{\frac{1}{2}\left[(\vec{\nabla} \varphi)(x)^{2}+m^{2} \varphi(x)^{2}\right]+g \varphi(x)^{r}\right\} d^{n} x$.

The affine story introduces an important new structure. To see that feature, we return to a single degree of freedom, with $p, q$. Our analysis instead focuses on the case where $q>0$. This case implies that the operator $P \neq P^{\dagger}$. We then seek an operator that can be self adjoint. The new classical variables are $p q, \ln (q)$, which, with $q>0$, permits each variable to be self adjoint. However, we also need to admit $q<0$, which can be done when we select unusual classical variables, namely, $p q, q$ which permits $-\infty<q \neq 0<\infty$, and joins together the cases where $q>0$ and $q<0$. Affine quantization then involves $p q \rightarrow\left(P^{\dagger} Q+Q P\right) / 2 \equiv D=D^{\dagger}$ and $q \rightarrow Q=Q^{\dagger}$, which obeys $[Q, D]=i \hbar Q$, and already offers a Lie algebra for the "affine group." Thus, we are led to the dilation operator $-\infty<D<\infty$ and the position operator $-\infty<Q \neq 0<\infty$. This analysis will implicitly be used in discussing the affine quantization of scalar fields.

For an affine field story we need $\pi(x) \varphi(x) \equiv \kappa(x)$ and $\varphi(x)$, for which $-\infty<\varphi(x) \neq 0<\infty$, and, as basic operators, $[\hat{\phi}(x), \hat{\kappa}(y)]=i \hbar \delta^{s}(x-y) \hat{\phi}(x)$. Just like the single degrees of freedom, we also have the classical relation that $\pi(x)^{2}=\kappa(x)^{2} / \varphi(x)^{2}$ as well. However, such a relation does not hold when quantized. In particular, for a single degree of freedom $D Q^{-2} D=P^{2}+(3 / 4) \hbar^{2} Q^{-2}$. For the scalar field, the analogous story leads to the relation that $\hat{\kappa}(x) \varphi(x)^{-2} \hat{\kappa}(x)=\hat{\pi}(x)^{2}+(3 / 4) \hbar^{2} \delta^{2 s}(0) \hat{\phi}(x)^{-2}$.

The new term involves a Dirac delta function, $\delta(0)=\infty$, raised to the power $2 s$. This new formal expression follows the similarly formal expression $\pi(x)^{2}=-\hbar^{2}\left[\delta^{2} / \delta \varphi(x)^{2}\right]$, wherein such formal terms require regularization, a procedure that we will introduce very soon. The latest relation is featured as an all important semi-classical relation in the following affine action 


$$
\begin{aligned}
S^{(a)}[\varphi]= & \int\left\{\frac{1}{2}\left[(\vec{\nabla} \varphi)(x)^{2}+m^{2} \varphi(x)^{2}\right]\right. \\
& \left.+g \varphi(x)^{r}+\frac{3}{8} \hbar^{2} \frac{\delta^{2 s}(0)}{\varphi(x)^{2}+\epsilon}\right\} d^{n} x,
\end{aligned}
$$

where $\epsilon$ is a regularization parameter.

\section{A. Lattice formulation of the field theory}

We used a lattice formulation of the field theory. The theory considers a real scalar field $\varphi$ taking the value $\varphi(x)$ on each site of a periodic, hypercubic, $n$-dimensional lattice of lattice spacing $a$ and periodicity $N a$. The affine action for the field, Eq. (7), is then approximated by

$$
\begin{aligned}
S^{(a)}[\varphi] / a^{n} \approx & \frac{1}{2}\left[\sum_{x, \mu} a^{-2}\left(\varphi(x)-\varphi\left(x+e_{\mu}\right)\right)^{2}+m^{2} \sum_{x} \varphi(x)^{2}\right] \\
& +\sum_{x} g \varphi(x)^{r}+\frac{3}{8} \sum_{x} \hbar^{2} \frac{a^{-2 s}}{\varphi(x)^{2}+\epsilon}
\end{aligned}
$$

where $e_{\mu}$ is a vector of length $a$ in the $+\mu$ direction.

In this paper we are interested in reaching the continuum limit by taking $N a$ fixed and letting $N \rightarrow \infty$.

\section{MONTE CARLO SIMULATION}

Our Monte Carlo (MC) simulations use the Metropolis algorithm $[10,11]$ to calculate the vacuum expectation of a functional observable $\mathcal{O}[\varphi]$

$$
\langle\mathcal{O}\rangle \approx \frac{\int \mathcal{O}[\varphi] \exp (-S[\varphi]) \prod_{x} d \varphi(x)}{\int \exp (-S[\varphi]) \prod_{x} d \varphi(x)},
$$

where $S=\int H d x_{0}$ is the action. This is a $N^{n}$ multidimensional integral. The simulation is started from the initial condition $\varphi=0$. One MC step consisted in a random displacement of each one of the $N^{n}$ components of $\varphi$ as $\varphi \rightarrow \varphi+(\eta-1 / 2) \delta$ where $\eta$ is a uniform pseudo random number in $[0,1]$ and $\delta$ is the amplitude of the displacement. Each one of these $N^{n}$ moves is accepted if $\exp (-\Delta S)>\eta$ where $\Delta S$ is the change in the action due to the move (it can be efficiently calculated considering how the kinetic part and the potential part change by the displacement of a single component of $\varphi$ ) and rejected otherwise. The amplitude $\delta$ is chosen in such a way to have acceptance ratios as close as possible to $1 / 2$ and is kept constant during the evolution of the simulation. One simulation consisted of $M=10^{6}$ steps. The statistical error on the average $\langle\mathcal{O}\rangle$ will then depend on the correlation time necessary to decorrelate the property $\mathcal{O}, \tau_{\mathcal{O}}$, and will be determined as $\sqrt{\tau_{\mathcal{O}} \sigma_{\mathcal{O}}^{2} /\left(M N^{n}\right)}$, where $\sigma_{\mathcal{O}}^{2}$ is the intrinsic variance for $\mathcal{O}$.
From the real field $\varphi(x)$ we extract the Fourier transform

$$
\tilde{\varphi}(p)=\int d^{n} x e^{i p \cdot x} \varphi(x),
$$

with $\tilde{\varphi}^{*}(p)=\tilde{\varphi}(-p)$. We then find the ensemble averages $\left\langle\tilde{\varphi}(0)^{2}\right\rangle$ and $\left\langle\tilde{\varphi}(0)^{4}\right\rangle$ and construct the following observable (a renormalized unitless coupling constant at zero momentum),

$$
g_{R}=\frac{3\left\langle\tilde{\varphi}(0)^{2}\right\rangle^{2}-\left\langle\tilde{\varphi}(0)^{4}\right\rangle}{\left\langle\tilde{\varphi}(0)^{2}\right\rangle^{2}},
$$

so that clearly, using path integrals in the Fourier transform of the field, we immediately find for the canonical version of the theory,

$$
g_{R} \stackrel{g \rightarrow 0}{\rightarrow} 0 .
$$

This remains true even for the calculation on a discrete lattice.

We then choose the momentum $p$ with one component equal to $2 \pi / N a$ and all other components zero and calculate the ensemble average $\left\langle|\tilde{\varphi}(p)|^{2}\right\rangle$. We then construct the renormalized mass

$$
m_{R}^{2}=\frac{p^{2}\left\langle|\tilde{\varphi}(p)|^{2}\right\rangle}{\left\langle\tilde{\varphi}(0)^{2}\right\rangle-\left\langle|\tilde{\varphi}(p)|^{2}\right\rangle} .
$$

When $g=0$ the canonical version of the theory can be solved exactly yielding

$$
m_{R} \stackrel{g \rightarrow 0}{\rightarrow}[\pi / N \sin (\pi / N)] m .
$$

Following Freedman et al. [3] we will call $g_{R}$ a dimensionless renormalized coupling constant and we will use it to test the "freedomness" of our field theories in the continuum limit. Note that the sum rules of Eqs. (12) and (14) do not hold for the affine version (7) of the field theory due to the additional $(3 / 8) \hbar^{2} \delta^{2 s}(0) /\left[\varphi(x)^{2}+\epsilon\right]$ interaction term.

\section{A. MC results}

In our MC simulation, following Freedman et al. [3], for each $N$ and $g$, we adjusted the bare mass $m$ in such a way to maintain the renormalized mass approximately constant $m_{R} \approx 3$ to within a few percent (in all cases less than $15 \%$ ), and we measured the renormalized coupling constant $g_{R}$ of Eq. (11) for various values of the bare coupling constant $g$ at a given small value of the lattice spacing $a=1 / N$. In order to keep the renormalized mass constant at large $g$, it was necessary to choose a negative $m^{2}$ giving rise to a double well interaction density. So that, with $N a$ and $m_{R}$ fixed, as $a$ was made smaller, whatever change we found in $g_{R} m_{R}^{n}$ as a function of $g$ could only be due to the change in 

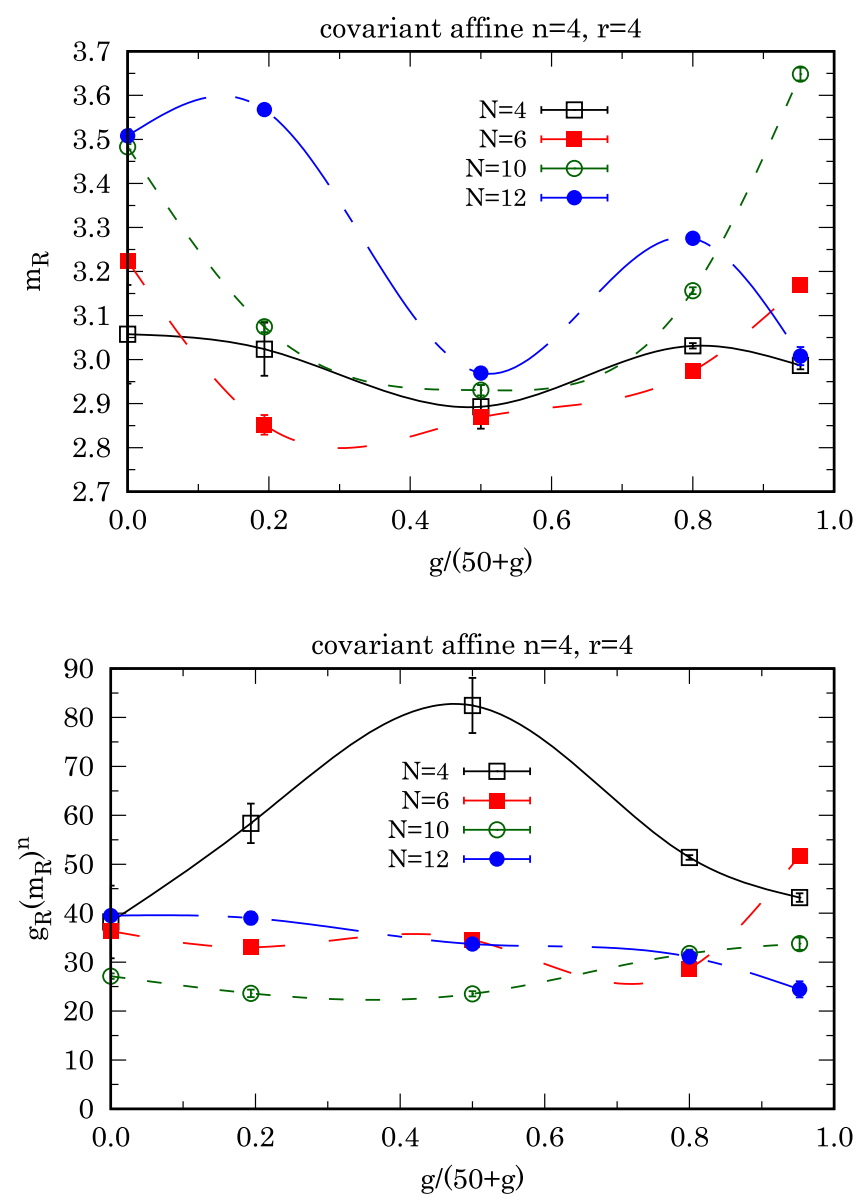

FIG. 1. We show the renormalized mass $m_{R}$ of Eq. (13) (top panel) and the renormalized coupling constant $g_{R} m_{R}^{n}$ of Eq. (11) (bottom panel) as calculated from Eq. (10) for $m_{R} \approx 3$ and various values of the bare coupling constant $g$ at decreasing values of the lattice spacing $a=1 / N(N \rightarrow \infty$ continuum limit) for the affine $\left(\varphi^{4}\right)_{4}$ Euclidean scalar field theory described by the action in Eq. (7). The lines connecting the simulation points are just a guide for the eye.

$a$. We generally found that a depression in $m_{R}$ produced an elevation in the corresponding value of $g_{R}$ and viceversa. The results are shown in Fig. 1 for the affine version with $\hbar=1$ and $\epsilon=10^{-10}$, where, following Freedman et al. [3] we decided to compress the range of $g$ for display, by choosing the horizontal axis to be $g /(50+g)$. As we can see from the top panel of the figure the renormalized mass was made to stay around the chosen value of 3 , even if this constraint was not easy to implement since for each $N$ and $g$ we had to run the simulation several (5-10) times with different values of the bare mass $m$.

These results should be compared with the results of Fig. 1 of Freedman et al. [3] where the same calculation was done for the canonical version of the field theory. As we can see from Fig. 1, contrary to the figure of Freedman, the renormalized coupling constant of the affine version remains far from zero in the continuum limit $(\mathrm{Na}=1$ and
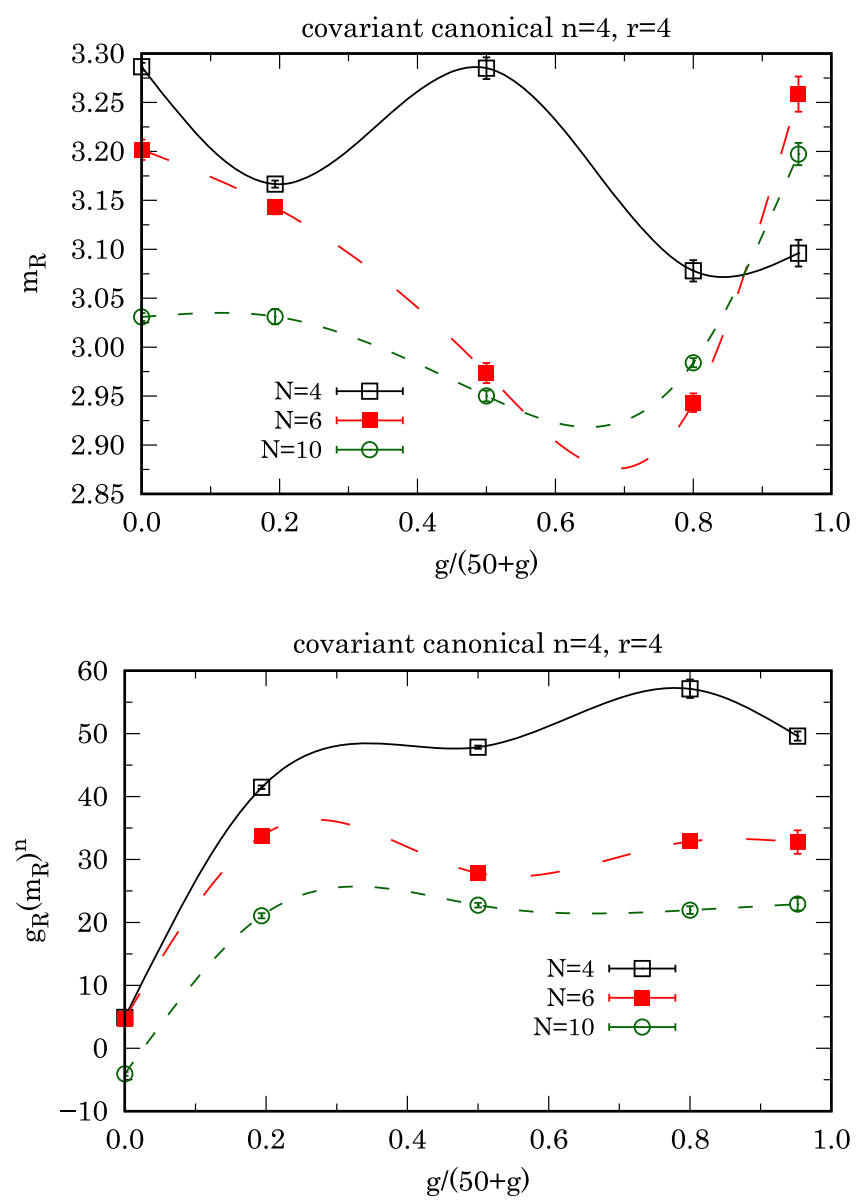

FIG. 2. We show the renormalized mass $m_{R}$ of Eq. (13) (top panel) and the renormalized coupling constant $g_{R} m_{R}^{n}$ of Eq. (11) (bottom panel) as calculated from Eq. (10) for $m_{R} \approx 3$ and various values of the bare coupling constant $g$ at decreasing values of the lattice spacing $a=1 / N(N \rightarrow \infty$ continuum limit) for the canonical $\left(\varphi^{4}\right)_{4}$ Euclidean scalar field theory described by the action in Eq. (6). The lines connecting the simulation points are just a guide for the eye.

$N \rightarrow \infty$ ) for all values of the bare coupling constant. Here, unlike in the canonical version used by Freedman, the diminishing space between higher $N$ curves is a pointer toward a nonfree ultimate behavior as $N \rightarrow \infty$. Moreover going from $N=10$ to $N=12$ we actually observe a growth in the renormalized coupling constant.

In order to test our calculations we repeated the Freedman calculation for the canonical version of the $\left(\varphi^{4}\right)_{4}$ theory, by keeping as before $m_{R} \approx 3$ (to within a $10 \%$ ) for all cases. Our results, shown in Fig. 2, compare favorably with the ones of Freedman Fig. 1.

\section{CONCLUSIONS}

The model on which this paper has focused is $\left(\varphi^{4}\right)_{4}$ and normally uses canonical quantization that was the only procedure, or other procedures designed to get equivalent results. For our model, canonical quantization leads to a 
free theory, as if the interaction term was missing. Affine quantization is a newer procedure that is qualitatively different than canonical quantization. The difference is such that favored Cartesian classical variables [12] choose the basic quantum operators, while for affine quantization, the favored classical variables arise from a constant negative curvature. This is different from the constant zero curvature, i.e., a flat surface suitable for canonical quantization. Indeed, it leads to affine quantization becoming a partner procedure and not a substitute for canonical quantization, with each procedure appropriate for distinct sets of problems for them to treat. This distinction of procedures offered by affine quantization has already clarified the analysis of half-harmonic oscillators [13], a favorable quantization of the nonrenormalizable model $\left(\varphi^{12}\right)_{3}$ [9], and now the strongest test yet for affine quantization, specifically, quantizing the $\left(\varphi^{4}\right)_{4}$ model.

It is expected that additional quantum examples may lead to problems when canonical quantization is used. Such examples deserve to consider affine quantization, which might be the answer to those problems.
[1] O. A. Ladyzenskaja, V. Solonnikov, and N. N. Ural'ceva, Linear and Quasi-Linear Equations of Parabolic Type (American Mathematical Society, Providence, 1968).

[2] J. R. Klauder, Beyond Conventional Quantization (Cambridge University Press, Cambridge, England, 2000), Chap. 5.

[3] B. Freedman, P. Smolensky, and D. Weingarten, Monte Carlo evaluation of the continuum limit of $\phi_{4}^{4}$ and $\phi_{3}^{4}$, Phys. Lett. 113B, 481 (1982).

[4] M. Aizenman, Proof of the Triviality of $\phi_{d}^{4}$ Field Theory and Some Mean-Field Features of Ising Models for $d>4$, Phys. Rev. Lett. 47, 886(E) (1981).

[5] J. Fröhlich, On the triviality of $\lambda \phi_{d}^{4}$ theories and the approach to the critical point in $d \geq 4$ dimensions, Nucl. Phys. B200, 281 (1982).

[6] J. Siefert and U. Wolff, Triviality of $\varphi^{4}$ theory in a finite volume scheme adapted to the broken phase, Phys. Lett. B 733, 11 (2014).
[7] J. R. Klauder, An ultralocal classical and quantum gravity theory, J. High Energy Phys., Gravitation Cosmol. 06 (2020) 656.

[8] J. R. Klauder, The benefits of affine quantization, J. High Energy Phys., Gravitation Cosmol. 06 (2020) 175.

[9] R. Fantoni, Monte Carlo evaluation of the continuum limit of $\left(\phi^{12}\right)_{3}$, arXiv:2011.09862.

[10] M. H. Kalos and P. A. Whitlock, Monte Carlo Methods (Wiley-Vch Verlag GmbH \& Co., Germany, 2008).

[11] N. Metropolis, A. W. Rosenbluth, M. N. Rosenbluth, A. M. Teller, and E. Teller, Equation of state calculations by fast computing machines, J. Chem. Phys. 21, 1087 (1953).

[12] P. A. M. Dirac, The Principles of Quantum Mechanics (Claredon Press, Oxford, 1958) in a footnote on page 114.

[13] L. Gouba, Affine quantization on the half line, J. High Energy Phys., Gravitation Cosmol. 07 (2021) 352. 